\title{
FORMACIÓN PERMANENTE DE LAS PERSONAS CON CAPACIDADES DIFERENTES EN TIEMPOS DE.PANDEMIA: SOCIALIZACIÓN Y APRENDIZAJE ALTERNATIVO EN EL ÁMBITO PROFESIONAL
}

\author{
Ángel Luis González Olivares \\ Universidad de Castilla - La Mancha \\ ALuis.Gonzalez@uclm.es
}

Recepción Artículo: 13 octubre 2021 Admisión Evaluación: 13 octubre 2021 Informe Evaluador 1: 13 octubre 2021

Informe Evaluador 2: 14 octubre 2021 Aprobación Publicación: 14 octubre 2021

\section{RESUMEN}

La COVID -19 ha modificado de manera muy rápida nuestra forma habitual de aprendizaje y también nuestra socialización debido principalmente al permutar el medio de base de la manera de comunicarse. Los roles de los protagonistas y participantes en la interacción recíproca han cambiado de funciones y actuaciones, porque se ha plasmado un rigor organizativo, exigido y deseado eficientemente en la didáctica y organización escolar de siempre, pero ahora priorizando y desplazando la espontaneidad y naturalidad, que la conversación habitual en los escenarios y contextos educativos tenían por simple hecho de estar y participar en ella. Ahora aprender es centrarse en buscar el motivo o interés de hacerlo, sustituyendo el desarrollo de las habilidades sociales como un recurso de ayuda, a ser la comunicación un sencillo medio de intercambiar intereses, alejándose de lo vicario y observacional, tan enriquecedor que ha sido para la enseñanza aprendizaje de las personas. En el colectivo de las personas con capacidades diferentes, tanto ellas como sus familias, han padecido estos cambios descritos y especialmente el distanciamiento social con las instituciones y con las personas ajenas a su núcleo familiar principal. Ha existido una importante limitación en el desarrollo de intervenciones terapéuticas, como en procesos y programas que ayudan a la integración social, principalmente centrados en el fomento de relaciones interpersonales. El presente estudio centrado en un proyecto de economía social de la entidad Almida se describen y detaIlan algunas de las consecuencias, temporales o definitivas, en las circunstancias personales de las personas afectadas con diferentes discapacidades, observando elementos y factores importantes que han influido en la preparación y cualificación profesional, así como en el empleo.

Palabras clave: formación permanente; capacidades diferentes: COVID-19 y educación; pandemia y educación 


\title{
FORMACIÓN PERMANENTE DE LAS PERSONAS CON CAPACIDADES DIFERENTES EN TIEMPOS DE PANDEMIA:
} SOCIALIZACIÓN Y APRENDIZAJE ALTERNATIVO EN EL ÁMBITO PROFESIONAL

\begin{abstract}
Permanent training of people with different capacities in times of pandemic: socialization and alternative learning in the professional field. COVID-19 has very quickly modified our usual way of learning and also our socialization, mainly due to the permuting of the basic means of communication. The roles of the protagonists and participants in the reciprocal interaction have changed functions and actions, because an organizational rigor has been reflected, efficiently demanded and desired in the didactics and school organization of always, but now prioritizing and displacing spontaneity and naturalness, which the usual conversation in educational settings and contexts had for the simple fact of being and participating in it. Now learning is to focus on looking for the reason or interest to do so, substituting the development of social skills as a helpful resource, to be communication a simple means of exchanging interests, moving away from the vicarious and observational, so enriching that it has been for teaching learning of people. In the group of people with different capacities, both they and their families have suffered these changes described and especially social distancing with institutions and with people outside their main family nucleus. There has been an important limitation in the development of therapeutic interventions, such as processes and programs that help social integration, mainly focused on promoting interpersonal relationships. This study focused on a social economy project of the Almida entity describes and details some of the consequences, temporary or definitive, in the personal circumstances of affected people with different disabilities, observing important elements and factors that have influenced the preparation and professional qualification, as well as in employment.
\end{abstract}

Keywords: permanent training; different capacities; COVID-19 and education; pandemic and education

\section{INTRODUCCIÓN}

El España en el primer trimestre del año 2020 apareció una enfermedad denominada "coronavirus infectious disease-19" (conocida con el acrónimo COVID-19: Corona Virus Disease 2019) como una enfermedad infectocontagiosa respiratoria, causada por "severe acute respiratory syndrome coronavirus-2" (SARS CoV-2). La propia Organización Mundial de la Salud (OMS) la clasificó como pandemia y lo trató con conjunto de recomendaciones de medidas políticas, sociales y sanitarias a todos los Estados y Naciones para afrontarlo.

Las medidas políticas, sociales y sanitarias se basaron en la prevención y el control epidemiológico de la COVID-19, causando un desconocido contexto socio-familiar con contradicciones psicológicas para la persona, la familia y la sociedad. El aislamiento y el distanciamiento social, provocadas por las medidas de confinamiento, limitaron los desplazamientos, la participación presencial en actividades y programas, de igual modo, las acciones socializadoras como cívicas, naturales de la pertenencia a sociedades y entidades culturales, educativas y terapéuticas. Como novedad y necesidad se iniciaron programas de participación a distancia y actuaciones telemáticas con el objetivo de permanecer y continuar con el avance en los aprendizajes, obteniendo consecuencias positivas, aunque diferentes y desconocidas, algunas negativas, con muchas contradictorias y retrospectivas.

Como históricamente ha ocurrido la sociedad y las personas que las forman reaccionan y cambian para responder a los problemas. En este caso se transformó a las nuevas maneras de organizarse, de interrelacionarse las personas, a las nuevas preferencias e intereses y, sobretodo, adaptándose a la innovación. Se consolidó, por circunstancias, una sociedad comunicada. Como describen Darretxe Urrutxi et al. (2020) el concepto de la sociedad red cambia el énfasis a la transformación organizativa y a la aparición de una estructura social globalmente interdependiente, con sus procesos de dominación y contra-dominación

Todo ello originó cambios en proyectos y programas sociales de atención e intervención en colectivos específicos, concretamente en los programas desarrollados en proyectos de Economía Social. Ámbito muy importante en el desarrollo económico y en la dinamización social de las personas e las instituciones sociales. La Resolución de 15 de marzo de 2018, de la Secretaría de Estado de Empleo, por la que se publicó el Acuerdo del Consejo de Ministros de 29 de diciembre de 2017, por el que se aprobó la Estrategia Española de Economía Social 20172020, razonaba que el sector de la economía social era un activo empresarial de primer nivel de la sociedad. En él se incluyen más de 43.000 empresas y entidades que, con independencia de la forma jurídica que adoptan, 
actúan conforme a los principios orientadores de la economía social (aplicación de los resultados obtenidos de la actividad económica principalmente en función del trabajo aportado y servicio o actividad realizada, promoción de la solidaridad interna y con la sociedad, e independencia respecto a los poderes públicos, entre otros). El Proyecto de Economía Social de Personas con Capacidades Diferentes Almida es uno de los referentes y en este estudio se conocerá qué cambios ha originado en él la pandemia.

\section{OBJETIVOS DE LA INVESTIGACIÓN Y SU CONTEXTO DE ESTUDIO}

La COVID 19 originó, desde el ámbito de la atención educación y social, múltiples asuntos a debate. Concretar a quién, dónde y cómo afectó y ha afectado con mayor ímpetu el paso de la atención, asistencia y enseñanza presencial a la modalidad virtual es algo incierto. Según las cifras del gobierno, el 25\% de la población en este periodo ha sufrido la enseñanza virtual de un modo u otro. Demuestra Cabrera (2020), que tanto al alumnado, como a la familia les ha afectado negativamente el cierre de centros y recursos. Casi un millón de personas participantes en programas les ha afectado por motivos personales y familiares.

El objetivo de esta investigación es conocer cómo afectó la pandemia en las relacionadas con la socialización de las personas más vulnerables, concretamente personas con capacidades diferentes, tanto desde el aspecto personal, como del familiar. Reconociendo que son muchos los años que desde las administraciones se ha intentado hacer que la educación sea mucho más inclusiva para aquellos que tiene una mayor dificultad en el aprendizaje. Pero nos cuestionamos si esa inclusión ha estado bien dirigida, o como dice Echeita (2020), ha sido una antigua integración. De esta manera lo afirman Del Barrio et al. (2005), que defienden que esta inclusión ha sido una, antiguamente llamada, integración. Para los participantes de programas educativos y formativos fue un mero cambio de domicilio, de centros de educación especial a centros de educación normalizada, ya que la docencia, es estos últimos, seguía siendo tal y como se venía haciendo habitualmente.

La adaptación de los medios y recursos, así como, la concienciación de los profesionales fue rapidísima, se compartía un espíritu de querer ayudar, considerando que la necesidad imperaba en todos los aspectos. En el afán de ayudar se preguntaba el cuándo y el cómo llegará el día en que asumamos con todas sus consecuencias y derivas la premisa de que todos tenemos, como describe Etxeberria (2018), la misma dignidad como personas y conllevando los mismos derechos a tener oportunidades iguales o equiparables para educarnos, trabajar, 0 disfrutar del ocio y la cultura en la común comunidad, así como para recibir igual atención, cuidados y el reconocimiento debido (Honneth, 1997). Esa preocupación lo argumenta Figueroa (2020) explicando que en pandemia emergen factores culturales que impregnan las relaciones sociales cotidianas y la percepción de las responsabilidades que se tienen sobre lo individual, Io familiar y lo social.

El contexto del estudio se centró en el Proyecto de Economía Social Almida, entidad especializada en el empleo protegido de personas con discapacidad. En sus programas la socialización se enmarca a partir de edades posteriores a la formación académica obligatoria, generalmente a partir de los 18 años. El alumnado que emprende su participación en otros escenarios y experiencias, dejando atrás su contacto ordinario diario con sus iguales que tenía en el centro educativo. Sus nuevos objetivos se dirigen a tener un empleo en el contexto social del mercado de trabajo, con oportunidades reales o con la participación en programas de preparación y cualificación profesional.

La entidad Almida desde su creación en el año 2005 ha desarrollado programas de orientación, formación y creación de empleo para ayudar y mejorar el aprendizaje personal y social del colectivo. Logrando la socialización e inclusión en situaciones y contextos ordinarios. Especialmente en empresas con la enseñanza de responsabilidades y comportamientos idóneos que favorecen la integración en la sociedad. Las acciones se han centrado, de manera presencial, en la planificación de procesos de adquisición de las habilidades sociales para ayudar a esa socialización. La COVID - 19 paró todos los programas en el estado de alarma declarado por el artículo 3 del Real Decreto 463/2020, de 14 de marzo. Y en sus consecuentes prórrogas se intentaron modificar con alternativas telemáticas las acciones para no retroceder en lo adquirido. 


\section{FORMACIÓN PERMANENTE DE LAS PERSONAS CON CAPACIDADES DIFERENTES EN TIEMPOS DE PANDEMIA: SOCIALIZACIÓN Y APRENDIZAJE ALTERNATIVO EN EL ÁMBITO PROFESIONAL}

Las actuaciones individualizadas por videoconferencias y videollamadas pretendieron dar continuidad a los programas grupales de formación que habían tenido mucho éxito en los espacios empresariales, pero perdía la esencia que identificaba la realidad vivida. Originando nuevas experiencias innovadoras y sorprendentes, pero también distintas. Creando desmotivación y desesperanza de no volver a lo vivido. Y con este proceso cambiando las expectativas positivas, que años habían sido trabajadas con éxito, por inseguridad que aún en nuestros días todavía perdura, porque los confinamientos y las medidas de prudencia, justificadas por recomendación sanitaria, secundan las oportunidades de este colectivo, añorando un sueño que una vez fue una realidad.

\section{LA ECONOMÍA SOCIAL Y SUS PARTICIPANTES}

La Economía Social en España está definida con la Ley 5/2011, de 29 de marzo, de Economía Social, que reconoce, visibiliza y desarrolla el sector. La Ley 31/2015, de 9 de septiembre, modifica y actualiza la normativa en materia de autoempleo y adopta medidas de fomento y promoción del trabajo autónomo y de la Economía Social, estableciendo medidas de desarrollo y fomento de la economía social española. Se define como un conjunto de actividades económicas y empresariales, sus principios persiguen el interés general económico o social.

Las entidades que constituyen la Economía Social son: cooperativas, sociedades laborales, mutualidades, centros especiales de empleo, empresas de inserción, las asociaciones vinculadas al movimiento de la discapacidad y de la inserción de personas en exclusión y las fundaciones de este ámbito. Para la Confederación Nacional de Centros Especiales de Empleo (CONACEE), estas entidades nacen con la Ley 13/1982, de 7 de abril, de integración social de los minusválidos, y el Real Decreto Legislativo 1/2013, de 29 de noviembre, por el que se aprueba el Texto Refundido de la Ley General de derechos de las personas con discapacidad y de su inclusión social, los define como entidades que tienen como objetivo realizar una actividad productiva de bienes o de servicios, participando regularmente en las operaciones del mercado, y tienen como finalidad el asegurar un empleo remunerado para las personas con discapacidad; a la vez que son un medio de inclusión del mayor número de estas personas en el régimen de empleo ordinario.

El Proyecto de Economía Social de Personas con Capacidades Diferentes Almida, es un Centro Especial de Empleo de la Junta de Comunidades de Castilla La Mancha, cuyo objetivo principal se centra en proporcionar a Ios trabajadores con discapacidad la realización de un trabajo productivo y remunerado, adecuado a sus características personales, facilitándoles una integración social y laboral en la sociedad. Las personas que forman parte de sus programas son 2850 personas, distribuidas de manera proporcional en las diferentes actividades (orientación, formación, empleo, ...)

\section{METODOLOGÍA DE ESTUDIO}

El periodo de pandemia y confinamiento ha producido modificaciones en el proceso de enseñanza-aprendizaje, como en las intervenciones y en los procedimientos terapéuticos, cambiando de un escenario presencial a otro virtual o telemático, motivo que ha provocado una reflexión y un análisis y evaluación de la manera de actuar. La atención telemática ha demostrado que es mejorable, principalmente en la profundización de los contenidos. Además, la desigualdad entre los participantes, alumnado y usuarios, no ha garantizado las mismas posibilidades para todos y todas. La adecuación en las tareas ha ido en perjuicio de la calidad (Aguilar, 2020) porque, no solo estar presente de manera virtual o participar, ha asegurado una misma certeza en los programas. Aun así, se debe comprender que al no haber elegido esta modalidad las consecuencias de no participación, nulo aprendizaje y escasa interacción, serían más problemáticas.

La metodología ha consistido en la observación y descripción de los procesos y programas de la entidad, comparando los resultados que existían antes del periodo de pandemia, durante la misma y la leve recuperación posterior a ella. Se ha considerado que este periodo nos has hecho aprender que el uso de la tecnología es vital en el campo de la educación (Ola, 2020). Y cuando llegue la normalización en el proceso de enseñanza-aprendizaje sería trascendente que se continuara con el uso de las tecnologías digitales que se hallen al alcance de todos 
como pizarras digitales, kahoot, zoom, classroom, etc.; porque proporcionan un aprendizaje mucho más autónomo. El uso de estas herramientas según Aguilar (2011) origina posturas del pensamiento diferentes: apreciación subjetiva de un hecho tecnológico; descripción objetiva de un proceso tecnológico; y los resultados, productos, metas y objetivos alcanzados. Por este motivo, en la docencia se tiene que hacer reflexionar al alumnado de la importancia que tiene el uso de estas herramientas para conseguir el cambio del sujeto y de la sociedad. Y el sector del aprendizaje considerar que no existe edad ni contexto, trasladando esta utilidad a otros escenarios como sociales y culturales, desarrollados por entidades de atención social, es un camino que proyecta un proceso que debe consolidarse.

El reto de los docentes no se limita únicamente a la enseñanza de contenidos, sino que debe atender emociones de los educandos, siendo una forma diferente de aprendizaje (Villanueva, 2020). Los contenidos que debe comunicar un docente tienen que partir desde una situación multidisciplinar y relacionando las condiciones actuales que viven el sujeto y su contexto. Con ello se podrá conseguir un equilibrio de las partes (educandoeducador), consiguiendo así generar un mayor índice de empatía, confianza o autonomía.

De igual manera, las familias han sido las aliadas en el control de las actitudes y comportamientos del aprendizaje, en el control de tiempo-espacio, y en las relaciones socioemocionales, creando y consolidando valores 0 nuevos estilos de aprendizaje. Sin ninguna duda, crear un buen ambiente familiar ha sido esencial.

Hay que recordar que la cultura se aprende en la familia, se complementa y mejora en el sistema educativo y, con la autonomía personal y la participación en otros contextos, llega a su desarrollo pleno de forma más particular. Aunque no siempre este proceso es tan fácil, hay predisposiciones, postulados y circunstancias que son análogas, pero también paradójicas. Responsabilidades, compromisos, méritos y desméritos se intercambian en Ios diferentes contextos, olvidando que el proceso de enseñanza-aprendizaje es algo muy global y complejo que se debe abordar en conjunto (González y Anguita, 2020).

\section{RESULTADOS CONOCIDOS Y UNA FORMA DIFERENTE DE ATENDER A LAS PERSONAS}

La situación de pandemia ha descrito que en la vulnerabilidad la afectación psicológica se acentuó en los colectivos específicos. Las reacciones psicológicas fueron diversas, según Terry - Jordán et al. (2020) las detaIlan como emocionales, cognitivas y conductuales. Autores como Urzúa et. al (2020) puntualizan que todos estos factores agravan la presencia de problemas de salud mental previos y propician y originan la aparición de nuevas problemáticas psicológicas, originando diagnósticos condicionados y tratamientos más dependientes. Por otro lado, Brooks et al. (2020) indagaron que el impacto psicológico durante la contingencia sanitaria ocasionó los cambios en la rutina habitual y la reducción del contacto social con los demás inducían aburrimiento, frustración y aislamiento, comportamientos inadecuados para los participantes. En su mayoría se hallaron efectos psicológicos perjudiciales, tales como síntomas de estrés postraumático, depresión, ansiedad, confusión y enojo (Alvites-Huamaní, 2020).

Por el contrario, hay algunas investigaciones que respaldan el convencimiento sobre la eficacia de las intervenciones basadas en la utilización del Internet (Andrews et al., 2010; Cárdenas et al., 2014; Ebert et al., 2018; Herrero et al., 2018; Pasarelu et al., 2017). Más específicamente en los escenarios de programas de intervención se pueden diferenciar tipologías de terapia por tele-asistencia: la terapia a distancia que se lleva a cabo vía videoconferencia y teléfono; la terapia web que hace uso de programas realizados sin apoyo de un terapeuta o profesional en tiempo real y, la terapia virtual que utiliza simulación de ambientes con o sin terapeuta o profesional de apoyo (González - Peña et al., 2017).

En los programas sociales de Almida, concretamente en los dirigidos para la orientación y asesoramiento a personas con capacidades diferentes, basados en el Real Decreto Legislativo 1/2013, de 29 de noviembre, por el que se aprueba el Texto Refundido de la Ley General de derechos de las personas con discapacidad y su inclusión social, y en el art. 43 informa que los Centros Especiales de Empleo deben prestar, a través de las unidades de apoyo, los servicios de ajuste personal y social que requieran las personas trabajadoras con discapacidad. Por 


\section{FORMACIÓN PERMANENTE DE LAS PERSONAS CON CAPACIDADES DIFERENTES EN TIEMPOS DE PANDEMIA: SOCIALIZACIÓN Y APRENDIZAJE ALTERNATIVO EN EL ÁMBITO PROFESIONAL}

lo que la entidad desarrolla programas de atención y asesoramiento para el empleo, orientando para la realización de un trabajo productivo, participando regularmente en las operaciones del mercado y teniendo como finalidad asegurar un empleo remunerado y la prestación de servicios de ajuste personal y social que requieran sus trabajadores con discapacidad, a la vez que es un medio de integración del mayor número de personas con discapacidad al régimen de trabajo normal. La referencia normativa es el Real Decreto 469/2006, de 21 de abril, que regula las unidades de apoyo a la actividad profesional en el marco de los servicios de ajuste personal y social de los Centros Especiales de Empleo.

El Almida el Programa de Mejora de las Competencias Sociocognitivas en Personas con Capacidades Diferentes, como conjunto de actuaciones de orientación social y laboral y asesoramiento para la consecución de competencias sociales, intelectuales y cognitivas, tiene la finalidad de mejorar la autonomía personal, la responsabilidad del proceso de aprendizaje personal y profesional, y las condiciones que favorecen la adquisición de competencias de afrontar retos y desafíos de la vida de cada persona con discapacidad. Este Programa se asienta en el paradigma cognitivo, en la orientación psicopedagógica y el aprendizaje significativo, y es una intervención con personas con discapacidad para ayudarles en su integración social y laboral. Está identificado con un enfoque ecológico, que defiende que la acción orientadora ha de plantearse desde las características y demandas del ambiente de cada persona, así como de las habilidades y destrezas funcionales necesarias para la adaptación del sujeto (González y Cruz, 2012; González-Olivares et. al., 2012; González y Beltrán, 2010).

Acciones que parten como centro la persona y su contexto. La interacción en él y, evidentemente, la participación presencial. El distanciamiento provocó la paralización y la modificación causó la incertidumbre y la ineficiencia de las intervenciones realizadas. Los efectos fueron las reacciones psicológicas adversas anteriormente comentadas, además del surgimiento de nuevas problemáticas psicológicas, atenuando agravamientos en los diagnósticos de los casos particulares, desmotivación y desinterés. La innovación tecnológica, justificada y necesaria para algunos casos, no fue idónea en este campo. La falta de recursos, la imperfecta familiarización del usuario o demora en la esperada intervención, determinaron la proyección llevada en los proyectos y programas de atención centrados en la intervención y la integración social.

\section{DISCUSIÓN Y CONCLUSIONES}

En el Proyecto de Economía Social de Personas con Capacidades Diferentes Almida, la pandemia detuvo proyectos con modelo de empleo inclusivo para este colectivo, como la Formación Junior (prácticas formativas, formación en contexto, mentoring...), las áreas sociales de especialización profesional en empresas ordinarias y el asesoramiento y coordinación de Proyectos de Empleo Inclusivo en contexto ordinario (empresas públicas y privadas).

En el estado de alarma los principales programas de Orientación y Asesoramiento para Personas con Capacidades Diferentes se interrumpieron e intentaron reanudarse de manera telemática, pero no tuvieron la validez programada. Algunos datos que pueden describir las consecuencias de lo sucedido se pueden observar a continuación.

El número de participantes en los programas han tenido la siguiente evolución: 
Gráfico 1: Elaboración propia.

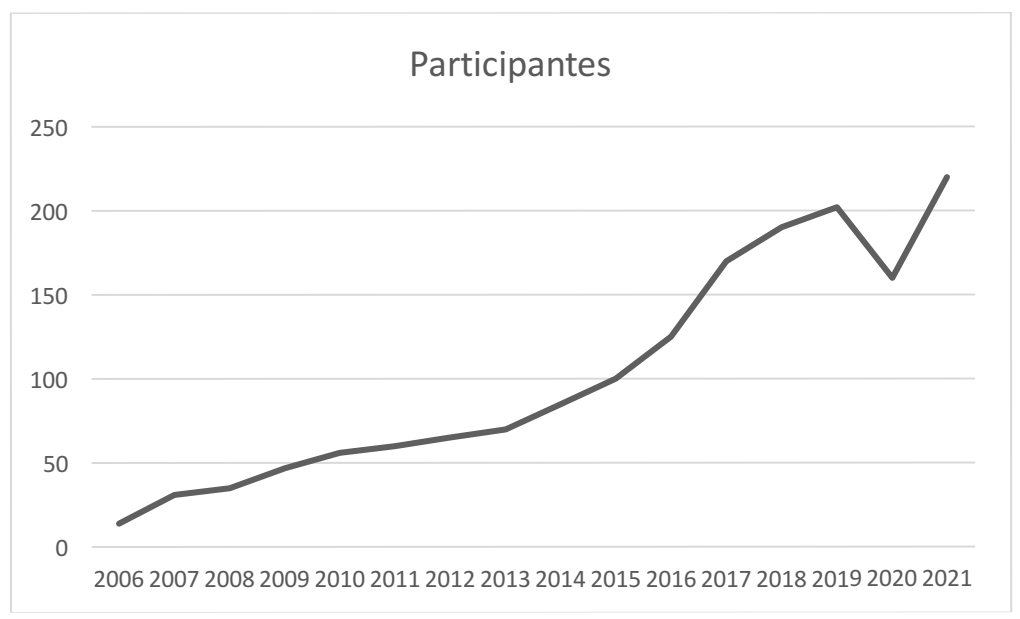

El progresivo crecimiento mantenido en los años anteriores ha sido afectado con un retroceso de participantes en el año 2020 con un 20\% de descenso, en el año 2021 todo ha mejorado, pero es destacable lo sucedido por la pandemia.

En relación a las acciones de intervención e inserción se puede contemplar lo siguiente:

Gráfico 2: Elaboración propia.

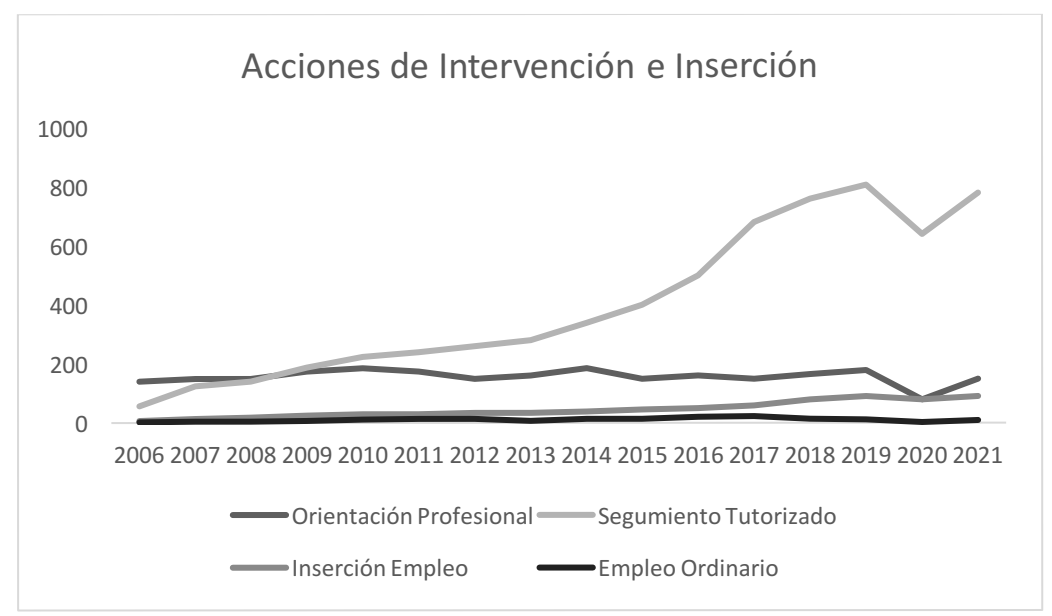

De manera más concreta el seguimiento tutorizado, dinamizador de las personas que se mantienen como participantes en los programas (2850 personas) conservaron la actividad, a pesar de su descenso por la situación. Las actuaciones de Orientación Profesional dirigidas a nuevos participantes descendieron en un 50\% y el empleo ordinario fue testimonial en el $1^{0}$ y $4^{0}$ trimestre, siendo totalmente nulo en el periodo de pandemia. 
En conclusión, la COVID -19 ha modificado de manera muy rápida nuestra forma habitual de aprendizaje y de igual manera nuestra socialización, debido al cambio de la manera de comunicarnos. El proceso de enseñanza-aprendizaje que se venía produciendo desde inicios del siglo XXI, con la utilización en las aulas de las nuevas tecnologías, ha acelerado su implantación. El rigor organizativo, exigido y querido eficientemente en la didáctica y organización escolar de siempre, pero ahora priorizando y desplazando la espontaneidad y naturalidad, que la conversación habitual en los escenarios y contextos educativos tenían por simple hecho de estar y participar en ella. Ahora aprender es centrarse en buscar el motivo o interés de hacerlo, sustituyendo el desarrollo de las habilidades sociales como un recurso de ayuda, a ser la comunicación un sencillo medio de intercambiar intereses, alejándose de lo vicario y observacional, tan enriquecedor que ha sido para la enseñanza aprendizaje de las personas. El 25\% de la población española, de una u otra manera ha sufrido las consecuencias de la pandemia por los cambios producidos en la educación. Desgraciadamente este hecho se ha sufrido de manera desigual, siendo los grupos sociales más vulnerables los que más lo han padecido. Los efectos en los proyectos y programas sociales de atención e intervención en colectivos específicos han sido considerables, suscitando nuevas experiencias innovadoras y sorprendentes, pero también distintas. Aunque, también, desmotivando y añorando experiencias vividas, propiciando miedo e incertidumbre.

En los Proyectos de Economía Social la pandemia ha tenido consecuencias, transitorias o irreversibles, con elementos y factores importantes, pero hay que mostrarse conforme que la sociedad está en constante cambio, evolucionando a nuevas organizaciones y prioridades, con diferentes particularidades e intereses, innovadoras y modernas, que requieren aprender, tener paciencia e ilusión, porque lo que venga y por lo que luchamos no es previsible cuando se desconoce, pero desearlo con expectativas negativas determina un recibimiento y una primera experiencia limitada, hecho que puede conllevar más problemática y un desánimo de toda esperanza.

\section{REFERENCIAS BIBLIOGRÁFICAS}

Aguilar, F. (2011). Reflexiones filosóficas sobre la tecnología y sus nuevos escenarios. Sophia. Colección de Filosofía de la Educación, (11), 123-174.

Aguilar, F. (2020). Del aprendizaje en escenarios presenciales al aprendizaje virtual en tiempos de pandemia. Estudios Pedagógicos XLVI, nํ3, 220-222.

Andrews, G., Cuijpers, P., Craske, M.G., McEvoy, P., \&Titov, N. (2010). Computer Therapy for the Anxiety and Depressive Disorders Is Effective, Acceptable and Practical Health Care: A Meta-Analysis. PLOS ONE, 5(10). https://doi.org/10.1371/journal.pone.0013196

Alvites-Huamaní, C. (2020). Covid-19: Pandemia que impacta en los estados de Ánimo. CienciAmérica, 9 (2). http://dx.doi.org/10.33210/ca.v9i2.327

Brooks, S. K., Webster, R. K., Smith, L. E., Woodland, L., Wessely, S., Greenberg, N., \& Rubin, G. J. (2020). The psychological impact of quarantine and how to reduce it: rapid review of the evidence. The Lancet, 395(10227), 912-920. https://doi.org/10.1016/S0140-6736(20)30460-8

Cabrera, L. (2020). Efectos del coronavirus en el sistema de enseñanza: aumenta la desigualdad de oportunidades educativas en España. Revista de Sociología de la Educación-RASE, 13 (2) Especial, COVID-19, 114-139. http://dx.doi.org/10.7203/RASE.13.2.17125.

Cárdenas, G., Botella, C., Quero, S., De la Rosa, A., y Baños, R. (2014). Programa de Telepsicología para el Tratamiento de la Fobia a hablar en público en Población Mexicana. Revista Psicología Iberoamericana, 22(1).

Darretxe Urrutxi, L., Beloki, B., \& Remiro, A. (2020). Sociedad y escuela que deseamos: La dialéctica entre inclusióny exclusión social. Ciencia y Educación, 4(1), 71-81. https://doi.org/10.22206/cyed.2020.v4i1.pp71-81

Del Barrio, C. Gutiérrez, H., Barrrios, A., van der Meulen, K y Granizo, L. (2005). Maltrato por abuso de poder entre escolares, ¿de qué estamos hablando? Revista Pediatría de Atención Primaria, 25 (7), $75-100$. 
Ebert, D., Van Daele, T., Nordgreen, T., Karekla, M., Compare, A., Zarbo, C., Brugnera, A., Øverland, S., Trebbi, G., Jensen, K. L., Kaehlke, F. \& Baumeister, H. (2018). Internet- and Mobile-Based Psychological Interventions: Appli cations, Efficacy, and Potential for Improving Mental Health. A Report of the EFPA EHealth Taskforce. European Psychologist, 23, 67-187. https://doi.org/10.1027/1016-9040/a000318

Echeita, G. (2020). La Pandemia del Covid-19. ¿Una oportunidad para pensar en cómo hacer más inclusivos nuestros sistemas educativos? Revista Internacional de Educación para la Justicia Social, 2020, 9 (1), 7-16.

Etxeberria, X. (2018). Ética de la inclusión y personas con discapacidad intelectual. Revista Española de Discapacidad, 6 (1), 281-290. https://doi.org/10.5569/2340-5104.06.01.14

Figueroa, J.G. (2020). Algunas reflexiones sobre dilemas éticos del cuidado en un entorno de pandemia. Psicologia \& Sociedade, 32, https://doi.org/10.1590/1807-0310/2020v32239528.

González, A.L. y Anguita, J. Mํ.. (2020) ¿Cómo afrontar la desigualdad y la exclusión social? En F.J. Hinojo, F. Sadio, J.A. López y J. M Romero, Experiencias e Investigaciones en Contextos Educativos (pp. 669-677). Dykinson.

González Olivares, A.L. y Cruz Martín-Serrano, R. (2012). Programa Almida. Programa de Mejora de las Competencias Sociocognitivas en Personas con Capacidades Diferentes. Acción Psicológica, 9 (2), pp. 5976. Doi: http://dx.doi.org/10.5944/ap.9.2.4105

González-Olivares, A.L., De-Juanas Oliva, A. y Diestro Fernández, A. (2012). Un Modelo Innovador de Aprendizaje en Personas con Discapacidad. Programa de Mejora de las Competencias Sociocognitivas en Personas con Capacidades Diferentes (Programa Almida). En Nieto, E., Callejas, A.I., Jerez, 0. (Coord.), Las competencias básicas. Competencias profesionales del docente. Ciudad Real: Universidad de Castilla La Mancha. pp. 901-910. ISBN: 978-84-694-3772-8. http://publicaciones.dipucres/tripascompetenciasdocente.pdf

González Olivares, A.L. y Beltrán Llera, J. (2010). Programa de mejora de las competencias sociocognitivas en personas con capacidades diferentes como recurso especializado en orientación. Revista de Psicología y $\begin{array}{llllll}\text { Educación, } \quad \text { Vol. } & 1, & \text { Núm. } & 5, & \text { p. } & 149-170 .\end{array}$ http://revistadepsicologiayeducacion.es/index.php/descargasj/finish/23/103.htm/

González-Peña, P., Torres, R., Del Barrio, V., y Olmedo, M. (2017). Uso de las nuevas tecnologías por parte de los psicólogos españoles y sus necesidades. Clínica y salud, 28, 81-91. https://doi.org/10.1016/j.clysa.2017.01.001

Herrero, R., Mira, A., Cormo, G., Etchemendy, E., Baños, R., García-Palacios, A., Ebert, D., Franke, M., Berger, T., Schaub, M., Görlich, Jacob, C. \& Botella, C. (2018). An Internet based intervention for improving resilience and coping strategies in university students: Study protocol for a randomized controlled trial. Internet Interventions. https://doi.org/10.1016/j.invent.2018.03.005

Honneth, A. (1997). La lucha por el reconocimiento. Crítica.

Ley 13/1982, de 7 de abril, de integración social de los minusválidos. BOE núm. 103, de 30 de abril de 1982, pp. 11106 a 11112.

Ley 5/2011, de 29 de marzo, de Economía Social, BOE núm. 76, de 30 de marzo de 2011, pp. 33023 a 33033.

Ley $31 / 2015$, de 9 de septiembre, por la que se modifica y actualiza la normativa en materia de autoempleo y se adoptan medidas de fomento y promoción del trabajo autónomo y de la Economía Social. BOE núm. 217, de 10 de septiembre de 2015, pp. 79824 a 79848.

Ola, A. L. (2020). Coronavirus: La deserción escolar es una amenaza pospandemia. https://forbescentroamerica.com/2020/06/30/coronavirus-la-desercion- escolar-es-una-amenaza-pospandemia/

Pasarelu, C., Andersson, G., Bergman, L., \& Dobrean, A. (2017). Internet-delivered transdiagnostic and tailored cognitive behavioral therapy for anxiety and depression: a systematic review and meta-analysis of randomized controlled trials. Cognitive Behaviour Therapy, 46(1), 1-28. https:// doi.org/10.1080/16506073.2016.1231219 
Real Decreto Legislativo 1/2013, de 29 de noviembre, por el que se aprueba el Texto Refundido de la Ley General de derechos de las personas con discapacidad y de su inclusión social, Pub. L. №. 1, 95635 (2013).

Real Decreto 469/2006, de 21 de abril, por el que se regulan las unidades de apoyo a la actividad profesional en el marco de los servicios de ajuste personal y social de los Centros Especiales de Empleo. BOE núm. 96, de 22 de abril de 2006, pp.15592 a 15596

Resolución de 15 de marzo de 2018, de la Secretaria de Estado de Empleo, por la que se publica el Acuerdo del Consejo de Ministros de 29 de diciembre de 2017, por el que se aprueba la Estrategia Española Social 2017 a 2020. BOE núm. 69, de 20 de marzo de 2018, pp. 31278 a 31313.

Terry-Jordán Y., Bravo-Hernández N., Elias-Armas KS, Espinosa-Caras I. (2020) Aspectos psicosociales asociados a la pandemia por COVID-19. Rev Inf Cient. 99(6):585-595. Disponible en: http://www.revinfcientifica.sld.cu/index.php/ric/article/view/3113

Urzúa A, Vera V.P., Caqueo U.A., Polanco C.R. (2020); La Psicología en la prevención y manejo del COVID-19. Aportes desde la evidencia inicial. Ter Psicol [en línea]. 38(1):103-118. Doi: http://dx.doi.org/10.4067/S0718-48082020000100103

Villanueva, L. (2020). El gran reto de la educación virtual en tiempos de pandemia. Fundación Wiese. Blog: https://www.fundacionwiese.org/blog/es/el-gran-reto-de-la-educacion-virtual-en-tiempos-de-pandemia/ 